\title{
A IMPORTÂNCIA DA SUPLEMENTAÇÃO PROTEICA NO PACIENTE BARIÁTRICO
}

\author{
Cristiane de Souza Pontes ${ }^{1}$ \\ Harley Correa Pereira ${ }^{2}$ \\ José Carlos de Sales Ferreira ${ }^{3}$
}

RESUMO: Introdução: Atualmente a cirurgia bariátrica é considerada a ferramenta mais eficaz no controle e no tratamento da obesidade severa. Os benefícios da cirurgia incluem resolução ou melhora acentuada de doenças crônicas como hipertensão, diabetes e hiperlipidemia. Entretanto, é preciso salientar que o tratamento cirúrgico da obesidade não se resume ao ato cirúrgico. Objetivo Geral: avaliar a importância da suplementação proteica no paciente bariátrico. Metodologia: Para a realização desse estudo optou-se por uma pesquisa na modalidade de revisão integrativa de literatura. A revisão integrativa determina o conhecimento atual sobre uma temática específica, já que é conduzida de modo a identificar, analisar e sintetizar resultados de estudos independentes sobre o mesmo assunto. Resultados e Discussão: Após a realização de uma cirurgia bariátrica, os pacientes sentem deficiência de proteína. Entre os macronutrientes necessários a fisiologia humana, depois desse procedimento, essa deficiência é a mais comumente relatada e isso ocorre por que, estimase que apenas $57 \%$ da proteína ingerida é absorvida após a gastroplastia. Conclusão: Ficou evidenciado que a cirurgia bariátrica representa atualmente a melhor estratégia para tratamento da obesidade mórbida. Entretanto, a rápida perda de peso que ocorre após a cirurgia vem acompanhada de uma considerável perda de massa muscular.

Palavras-chave: Suplementação, Bariátricos, Obesidade.

ABSTRACT: Introduction: Currently, bariatric surgery is considered the most effective tool in the control and treatment of severe obesity. The benefits of surgery include resolution or marked improvement of chronic diseases such as hypertension, diabetes and hyperlipidemia. However, it should be noted that the surgical treatment of obesity is not limited to the surgical act. General objective: to evaluate the importance of protein supplementation in bariatric patients. Methodology: In order to carry out this study, we opted for a research in the modality of integrative literature review. The integrative review determines the current knowledge on a specific theme, since it is conducted in order to identify, analyze and synthesize results of independent studies on the same subject. Results and Discussion: After undergoing bariatric surgery, patients experience protein deficiency. Among the macronutrients necessary for human physiology, after this procedure, this

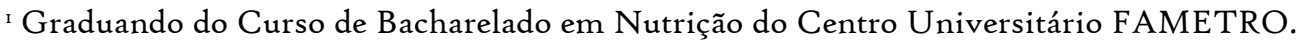

2 Graduando do Curso de Bacharelado em Nutrição do Centro Universitário FAMETRO. E-mail: harley.pereira@hotmail.com

${ }_{3}$ Orientador do TCC, Mestre em Ciência de Alimentos pela Universidade Federal do Amazonas.
} 
deficiency is the most commonly reported and this is because, it is estimated that only $57 \%$ of the protein ingested is absorbed after gastroplasty. Conclusion: It was evidenced that bariatric surgery currently represents the best strategy for the treatment of morbid obesity. However, the rapid weight loss that occurs after surgery is accompanied by considerable loss of muscle mass.

Keyword: Supplementation, Bariatric, Obesity.

\section{INTRODUÇÃO}

Sendo a obesidade uma condição médica crônica de etiologia multifatorial, o seu tratamento envolve várias abordagens (nutricional, uso de medicamentos antiobesidade e prática de exercícios físicos). Entretanto, vários pacientes não respondem a estas manobras terapêuticas, necessitando de uma intervenção mais eficaz. A cirurgia bariátrica tem se mostrado uma técnica de grande auxílio na condução clínica de alguns casos de obesidade. A indicação desta intervenção vem crescendo nos dias atuais e baseia-se numa análise abrangente de múltiplos aspectos do paciente (SEGAL; FANDIÑO, 2016; FAÉ; LIBERALI; COUTINHO, 2015).

Atualmente a cirurgia bariátrica é considerada a ferramenta mais eficaz no controle e no tratamento da obesidade severa. Os benefícios da cirurgia incluem resolução ou melhora acentuada de doenças crônicas como hipertensão, diabetes e hiperlipidemia. Entretanto, é preciso salientar que o tratamento cirúrgico da obesidade não se resume ao ato cirúrgico (FARIA; CARDEAL, 2014; MAGNO et al., 2014).

Estudos in vivo têm demonstrado que algumas deficiências nutricionais como vitamina $\mathrm{D}_{2-4}$, vitamina A 5,6 e zinco estão concomitantemente presentes na obesidade, possivelmente por exercerem papel importante na regulação da adiposidade ou nos mecanismos de regulação do apetite. Dessa forma, é essencial que haja uma programação criteriosa para o acompanhamento clínico-nutricional. Tanto o pré quanto o pós operatório, imediato e tardio, são de extrema importância para o sucesso do tratamento. Nesse contexto, a frase "perda de peso de forma saudável" tem sido bem-empregada (MOREIRA; ESPÍNOLA; AZEVEDO, 2015; SILVA; GOMES, 2015).

As implicações dos procedimentos de cirurgia bariátrica no estado nutricional do paciente se devem especificamente às alterações anatômicas e fisiológicas que prejudicam as 
vias de absorção e/ou ingestão alimentar. Uma boa compreensão da fisiologia de absorção do trato gastrointestinal é muito importante para compreender as potenciais deficiências nutricionais após a cirurgia (CAMBI; BARETA; 2018; DELGADO et al., 2015).

O principal tipo de cirurgia bariátrica realizado atualmente é o bypass gástrico em Yde-Roux (BGYR), uma técnica cirúrgica mista por restringir o tamanho da cavidade gástrica e, consequentemente, a quantidade de alimentos ingerida, e por reduzir a superfície intestinal em contato com o alimento (disabsorção). A má absorção de nutrientes é umas das explicações para a perda de peso alcançada com o uso de técnicas disabsortivas como a derivação biliopancreática/duodenal switch (DBP), sendo que cerca de $25 \%$ de proteína e 72\% de gordura deixam de ser absorvidos (APPOLINARO, 2016).

Automaticamente, nutrientes que dependem da gordura dietética para serem absorvidos, como as vitaminas lipossolúveis e o zinco, estão mais suscetíveis a uma má absorção nesse tipo de procedimento. Vitaminas e minerais são fatores e cofatores essenciais em muitos processos biológicos que regulam o peso corporal direta ou indiretamente (KARIMI, 2017).

Os benefícios metabólicos desses micronutrientes no controle da perda de peso incluem a regulação do apetite, da fome, da absorção de nutrientes, da taxa metabólica, do metabolismo de lipídios e carboidratos, das funções das glândulas tireoide e suprarrenais, do armazenamento de energia, da homeostase da glicose, de atividades neurais, entre outros. Assim, a "adequação" de micronutrientes é importante não só para a manutenção da saúde, mas também para obter o máximo sucesso na manutenção e na perda de peso a longo prazo (CAPELLA, 2016).

Sabe-se que atualmente a cirurgia bariátrica é considerada a ferramenta mais eficaz no controle e no tratamento da obesidade severa. Os benefícios da cirurgia incluem resolução ou melhora acentuada de doenças crônicas como hipertensão, diabetes e hiperlipidemiar. Entretanto, é preciso salientar que o tratamento cirúrgico da obesidade não se resume ao ato cirúrgico, necessitando também do aporte nutricional adequado (BORDALLO et al., 2016).

Com isso, nota-se que a adequação alimentar para o caso proposto é essencial, tendo em vista que no caso da suplementação proteica em pacientes bariátricos há um enfeito a 
curto e longo prazo benéficos para a evolução do mesmo, sendo possível assim, atingir o objetivo proposto inicialmente, com a realização da cirurgia e a adequação no estilo de vida, justificando-se assim, o desenvolvimento desse estudo. Com o objetivo de avaliar a importância da suplementação proteica no paciente bariátrico.

\section{METOdOLOGIA}

\section{I Tipo de Estudo}

Para a realização desse estudo optou-se por uma pesquisa na modalidade de revisão integrativa de literatura. A revisão integrativa determina o conhecimento atual sobre uma temática específica, já que é conduzida de modo a identificar, analisar e sintetizar resultados de estudos independentes sobre o mesmo assunto (SILVEIRA et al., 2008).

A Revisão Integrativa é um método de pesquisa apontado como ferramenta de grande relevância no campo da saúde, por proporcionar a busca, a avaliação crítica e a síntese de evidências sobre um tema investigado. Esses aspectos facilitam a identificação dos resultados relevantes, de lacunas que direcionam para o desenvolvimento de futuras pesquisas e auxiliam o profissional a escolher condutas e a tomar decisões, proporcionando um saber crítico (SOUZA; SILVA; CARVALHO, 2оIо).

\subsection{Coleta de Dados}

Para extrair os dados dos artigos selecionados, será necessária a utilização de um instrumento previamente elaborado capaz de assegurar que a totalidade dos dados relevantes seja extraída, minimizar o risco de erros na transcrição, garantir precisão na checagem das informações e servir como registro (SOUZA; SILVA; CARVALHO, 2010).

A coleta das informações para a pesquisa bibliográfica será por meio da exploração da base de dados da Biblioteca Virtual em Saúde (BVS), Biblioteca Cientifica Eletrônica Virtual(SCIELO) e Literatura Latino-Americana e do Caribe em Ciências da Saúde(LILACS).

A busca na base de dados será orientada pelos descritores: Suplementação, Bariátricos, Obesidade, e será realizado em todos os índices, buscando captar o maior 
número de artigos publicados no período proposto que abordem a temática em discussão, visando o desenvolvimento fidedigno desse estudo.

\subsection{Análise de Dados}

Para uma análise crítica e reflexiva dos estudos incluídos na revisão, será realizada uma leitura minuciosa e criteriosa destacando os que atingirem os critérios de inclusão e que contemplarem o objetivo proposto, para viabilizar o resultado da pesquisa de forma objetiva.

Serão incluídos os artigos publicados em língua portuguesa nos últimos dez anos, com texto completo, disponível on-line, com acesso livre. Serão excluídos da amostra os artigos publicados em línguas estrangeiras, os que não apresentarem o texto na integra, artigos que não apresentavam relação direta com o tema, resumos, monografias, dissertações, teses e artigos repetidos.

\section{RESULTADOS E DISCUSSÃO}

3.I Os benefícios e possíveis consequências da cirurgia bariátrica

A cirurgia bariátrica é um procedimento voltado à perda de peso. Existem diversos tipos de cirurgia e a escolha do método mais adequado depende de algumas características do paciente. É extremamente importante lembrar que é um procedimento cirúrgico e deve ser uma decisão médica, e não apenas estética (PORIES, 2015).

Segundo o Ministério da Saúde, para ser candidata à cirurgia, a pessoa deve tentar por pelo menos dois anos os métodos tradicionais de emagrecimento. Isso inclui reeducação alimentar, tratamento psicológico, atividade física e uso de medicamentos em casos específicos. Se nenhuma dessas alternativas trouxer uma resposta positiva, ela poderá ser avaliada e então fará todos os exames necessários para verificar a possibilidade de ser operada (ADAMI, 2016).

Quanto aos riscos e benefícios, vale lembrar que se trata de um procedimento cirúrgico e, portanto, sempre haverá riscos. "Com a melhora dos materiais e aumento das habilidades manuais dos cirurgiões, as taxas de complicações agudas são bastante baixas atualmente", comenta o especialista (HSU, 2016). 
Vale lembrar alguns mitos inerentes à cirurgia bariátrica, como, por exemplo, a mulher não poder engravidar após sua realização. O ideal é esperar por dois anos, tempo que ainda está perdendo peso", esclarece o gastroenterologista. Outra dúvida constante é a obrigação de submeter-se a cirurgias plásticas para a retirada de pele após o procedimento, nem sempre há essa necessidade, pois depende do tamanho do emagrecimento, idade do paciente e até do tipo de pele (YUSUF, 2017).

O emagrecimento de forma consciente traz uma melhora importante na qualidade de vida. Ele diminui de maneira consistente a ocorrência de doenças, como hipertensão arterial, diabetes Mellitus tipo II, doenças do coração, AVCs, depressão, infertilidade e alguns tipos de câncer. De modo geral, após a cirurgia, é muito importante comparecer aos retornos médicos agendados. Além de respeitar a dieta imposta pela equipe cirúrgica e, sobretudo, saber que é um procedimento grande e a conversa com seu médico deve ser levada à risca (BONNE, 2018).

\subsection{A alimentação proteica: seus efeitos e indicações}

As proteínas, ao lado dos carboidratos e das gorduras, são um macronutriente que deve ser consumido diariamente para atender às necessidades do corpo. A ingestão dietética de referência para proteínas é de o,8 gramas por quilograma de peso. No entanto, muitas evidências sustentam a ideia de que uma maior ingestão de proteínas age auxiliando a perda de peso, além de apresentar outros benefícios à saúde (BRANDER, 2019).

No corpo, as proteínas desempenham as funções de reparo de músculos, ossos, pele e cabelo, participam na formação de hormônios e enzimas e no transporte e armazenamento de importantes moléculas, entre outras funções. A molécula de proteína é composta de unidades menores conhecidas como aminoácidos. Dos 22 aminoácidos existentes, 9 são considerados "aminoácidos essenciais", o que significa que são absolutamente necessários ao funcionamento do corpo. Nosso corpo não consegue produzi-los e eles devem ser obtidos através da alimentação (PUTTINI, 20I4).

É muito comum ver atletas que investem em alimentos proteicos ou suplementos de qualidade que possam garantir a quantidade necessária de proteína para sua dieta. É 
extremamente importante entender que os alimentos proteicos de uma maneira completa, são aqueles que possuem os aminoácidos essenciais (PIEGAS, 2015).

A dieta hiperproteica é aquela em que há um maior consumo de proteínas e gorduras, aliado a uma menor ingestão diária de carboidratos. A dieta hiperproteica prioriza carnes, laticínios e verduras, e diminui ou exclui do cardápio massas, pães, doces, a maioria das frutas e outras fontes de carboidratos. Com isso, ela faz emagrecer rapidamente, sobretudo nos primeiros dias (HOSS, 2019).

Aumentar a ingestão de proteínas diminui o apetite e o peso corporal e tem efeitos benéficos (aumento) sobre a taxa metabólica e composição corporal. Ingerir mais proteína pode ajudar a retardar a fome e aumentar a produção dos hormônios que ajudam a pessoa a se sentir saciada. Além disso, reduz os níveis de grelina, o "hormônio da fome" (CARVALHO, 2016).

\subsection{Os benefícios da suplementação proteica para o paciente bariátrico}

Após a realização de uma cirurgia bariátrica, os pacientes sentem deficiência de proteína. Entre os macronutrientes necessários a fisiologia humana, depois desse procedimento, essa deficiência é a mais comumente relatada e isso ocorre por que, estimase que apenas $57 \%$ da proteína ingerida é absorvidas após a gastroplastia (SEGAL, 2016).

Essa é uma consequência de deve ser acompanhada e tratada para evitar futuros problemas. Pós operados, os pacientes devem utilizar suplementos protéicos em pó e de alto valor biológico, sseguindo uma dieta hiperproteica. É recomendado o consumo de 20 gramas de proteína, três vezes ao dia, logo na primeira semana após a cirurgia. Após a segunda semana, recomendação de proteínas é de 120 gramas ao dia (MOLEIRO; FERRO; FERREIRA, 2019).

A desnutrição proteica, após a realização da cirurgia bariátrica, é a complicação mais grave e mais comum entre os pacientes. Diante desse quadro, outros especialistas têm recomendado $70 \mathrm{~g} /$ dia de proteína, durante restrições calóricas para a perda de peso. Porém, muitos programas de cirurgia bariátrica recomendam 60 a $80 \mathrm{~g} /$ dia de proteína ou I,o a I, 5 $\mathrm{g} / \mathrm{kg}$ de peso ideal, embora as necessidades exatas ainda não estejam definidas. Em estudos 
modernos, tem se observado que pacientes com dieta controlada com maior aporte proteico, perdem peso de forma mais rápida com maior perda de porcentagem de gordura (SABISTON, 2019).

Além do mais, a dieta reduzida em proteínas pode inibir a cicatrização da ferida cirúrgica, pois as proteínas são quebradas em aminoácidos e peptídeos que são necessários para a reparação de tecidos, tais como o colágeno. A quantidade de proteína necessária para essa reconstituição depende da idade do paciente, o tamanho da ferida e de fatores como o stress e as infecções presentes no quadro. Como a proteína tem a capacidade de contribuir para a síntese do colágeno e para a remodelagem da ferida, devemos ter na dieta alguns aminoácidos importantes como, Glutamina, Ornitina e Arginina e até mesmo o whey protein que é um suplemento que supre quando em doses certas a necessidade proteica (SUPLICY, 2015).

A suplementação é fundamental no pós operatório da cirurgia bariátrica mas, como em qualquer contexto, o uso desses elementos deve ser indicado por um profissional nutricionista ou pelo médico, que levarão em conta as necessidades individuais e a capacidade de ingestão alimentar. Utilizar suplemento sem indicação pode fazer mal à saúde. Um cardápio balanceado é aquele que regula a homeostase do organismo segundo a sua individualidade bioquímica, por isso que ele é sempre único e pessoal (REPETTO, 2017).

\section{${ }_{4}$ CONCLUSÃO}

Ficou evidenciado que a cirurgia bariátrica representa atualmente a melhor estratégia para tratamento da obesidade mórbida. Entretanto, a rápida perda de peso que ocorre após a cirurgia vem acompanhada de uma considerável perda de massa muscular. É importante destacar que a necessidade proteica no período pós-cirúrgico se torna ainda maior, pois há maior catabolismo.

No entanto, infelizmente, muitos pacientes não priorizam as proteínas em suas refeições, seja pela maior dificuldade de mastigação (principalmente as carnes) ou pela saciedade que esse macro nutriente produz. Sendo assim, fica evidenciado através de estudos que a melhor opção para atingirmos esta recomendação, é o uso de suplementos proteicos, 
pois a quantidade de proteína ingerida só através das refeições é muito baixa, principalmente nos primeiros 6 meses de pós-operatório.

\section{REFERENNCIAS}

ADAMI, G.F.; GANDOLFO, P.; BAUER.; et al. Binge eating in massively obese patients undergoing bariatric surgery. Int J of Eat Disords, v.3, n.17, p.45-50, 2016.

ALVES LFA, GONÇALVES RM, CORDEIRO GV. Beribéri pós bypass gástrico: uma complicação não tão rara. Relato de dois casos e revisão da literatura. Arq Bras Endocrinol Metab. 2006; 50:564-8.

ALWASAIDI, T. A.; ALAHMADI, D. K.; ALRUFAYI, B. M.; et al. Determining the prevalence and causes of anaemia in patients after bariatric surgery in a Saudi hospital. Journal of Taibah University Medical Sciences, v.15, n. 2, p.129-135, 2020.

BONNE, O.; BASHI, R.; BERRY, E. Anorexia Nervosa Following Gastroplasty in the Male: Two Cases. Intl. J of Eat Disord, v.6, n.19, p.105-108, 2018.

BORDALO, L.A.; MOURÃO, D.M.; BRESSAN, J. Deficiências nutricionais após cirurgia bariátrica, Acta. Med. Port., v.24, n.4, p. I02I-28, 201 .

BRANDER, Nathaniel. Autoestima e os seus seis pilares. São Paulo: Saraiva; 2017.

BOTELLA-CARRETERO JI, ALVAREZ-BLASCO F, VILLAFRUELA JJ. Vitamin D deficiency is associated with the metabolic syndrome in morbid obesity. Clin Nutr. 2017; 26(5):573-80.

BUCHWALD H, AVIDOR Y, BRAUNWALD E, et al. Bariatric surgery: a systematic review and metaanalysis. JAMA. 2014; 292(14):1724-37.

CAMBI, M.P.C.; BARETTA, G.A.P. Guia alimentar bariátrico: modelo do prato para pacientes submetidos à cirurgia bariátrica. $\mathrm{ABCD}$, arq. bras. cir. dig., v.31, n.2, p.124-30, 2018.

CARVALHO, M.C.V.S.; LUZ, M.T. Simbolismo sobre "natural" na alimentação. Cien Saude Colet., v.ı6, n.I, p.147-154, 2016

CARVALHO, A.D.A.S.; ROSA, R. D. S. Cirurgias bariátricas realizadas pelo Sistema Único de Saúde no período 2010-2016: estudo descritivo das hospitalizações no Brasil. Epidemiologia e Servicos de Saude: Revista Do Sistema Unico de Saude Do Brasil, v.28, n.I, p.I-II, 2019.

CARON, M.; HOULD, F.S.; LESCELLEUR, O.; et al. Long-term nutritional impact of sleeve gastrectomy. Surgery for Obesity and Related Diseases, v.13, n.10, p. 1664- 1673, 2017.

CASTANHA, C. R.; CASTANHA, A. R.; et al. Avaliação da qualidade de vida, perda de peso e comorbidades de pacientes submetidos à cirurgia bariátrica. Revista do Colégio Brasileiro de Cirurgiões, v.45, n.3, p.I-9, 2018.

COSTA, Fernando Vieira, et al. Anemias and nutritional deficiencies in patients undergoing gastrectomy: an integrative. Review. Research, Society and Development, v. 9, n.9, p.130-137, 2020.

DE LUCA, M.; ANGRISANI, L.; HIMPENSJ, A.J.; et al. Indications for surgery for obesity and weight- 
related diseases: position statements from the internacional federation for the surgery of obesity and metabolic disorders (IFSO). Obes Surg., v.4, n.26, p.1659 - I696, 2016.

DELGADO, Felippe Prado, et al. Effects of a multidisciplinary program on morbid obese patients and patients with comorbility who are likely to be candidates for bariatric surgery. Nutr Hosp., v.I, n.31, p.201-6, 2015.

DIXON JB, DIXON ME, OBRIEN PE. Elevated homocysteine levels with weight loss after Lap-Band surgery: higher folate and vitamin $\mathrm{B}_{12}$ levels required to maintain homocysteine level. Int J Obes Relat Metab Disord. 2001; 25(2):219-27.

FAÉ, C.; LIBERALI, R.; COUTINHO, V.F. Deficiência de nutrientes a longo prazo no pós-operatório de cirurgia bariátrica - revisão sistemática. Sa Bios: Rev. Saúde e Biol., v.1o, n.2, p. 46-53, 2015.

FARIA, S.L.; FARIA, O.P.; CARDEAL, M.A. Comparison of weight loss, food consumption and frequency of vomiting among Roux-en-Y gastric bypass patients with or without constriction ring. ABCD, arq. bras. cir. dig., v.27, n.I, p.43-46, 2014 .

FLORES, L.; MOIZÉ, V.; PUJOL, J.; et al. Prospective study of individualized or high fixed doses of vitamin D supplementation after bariatric surgery. ObesSurg., n.2, v.25, p.470-476, 2015.

GARRIDO JÚNIOR, Antônio Barros. Cirurgia da obesidade. São Paulo: Atheneu; 2016.

GESQUIERE, I.; FOULON, V.; AUGUSTIJNS, P.; et al. Micronutrient intake, from diet and supplements, and association with status markers in pre and post-RYGB patients.ClinNutr., v.3, n.23, p.26I- 4, 2016.

HANSEN, T.T.; JAKOBSEN, T.A.; NIELSEN, M.S.; et al. Hedonic changes in food choices following Roux-en-Y gastric bypass. ObesSurg., v.3, n.26, p. 1946 - 1955, 2016.

HOMAN, J.; SCHIJNS, W.; AARTS, E. O.; et al. An optimized multivitamin supplement lowers the number of vitamin and mineral deficiencies thre e years after Roux-en-Y gastric bypass: a [ cohort study. Surgery for Obesity and Related Diseases, v.12, n.3, p.659-667, 2016.

HO T, et al. Long-term results after laparoscopic roux-en-Y gastric bypass: ro-year follow-up. Surg Obes Relat Dis 2009; 5:Abstract PL-2II.

HSU, L.K.; BETANCOURT, S.; SULLIVAN, S.P. Eating Disturbances Before and After Vertical Banded Gastroplasty: A Pilot Study .Int J of Eat Disord, v.5, n.19, p. 23-34, 2016.

KIM, Y.W.; BAE, J.M.; PARK, Y.K.; et al. Effect of Intravenous Ferric Carboxymaltose on Hemoglobin Response Among Patients With Acute Isovolemic Anemia Following Gastrectomy: The FAIRY Randomized Clinical Trial. JAMA, v.31, n.20, p. 2097-2104, 2015.

KUMPF VJ, SLOCUM K, BINKLEY J. Complications after bariatric surgery: survey evaluating impact on the practice of specialized nutrition support. Nutr Clin Pract. 2007; 22(6):673-8.

LEDOUX S, MSIKA S, MOUSSA F, et al. Comparison of nutritional consequences of conventional therapy of obesity, adjustable gastric banding, and gastric bypass. Obes Surg. 2016; 16(8):1041-9.

LIN L.; ALLEMEKINDERS, H.; DANSBY, A., et al. Evidence of health benefits of canola oil. Nutr Rev. v.71, n.6, p.370-85, 2013. 
LOPES, G.D.; MOEHLECKE, M.; LOPES, S.F, et al. Whey Protein Supplementation Enhances Body Fat and Weight Loss in Women Long AfterBariatric Surgery: a Randomized Controlled Trial. ObesSurg., v.27, n.2, p. 424-431, 2017 .

LOSS, A.B.; SOUZA, A.A.P.; PITOMBO, C.A., et al. Avaliação da síndrome de dumping em pacientes obesos mórbidos submetidos à operação de bypass gástrico com reconstrução em Y de Roux. Rev Col Bras Cir., v.36, n.5, p.413-419, 2019.

LUPOLI, R.; LEMBO, E.; SALDALAMACCHIA, G. Bariatric surgery and long-term nutritional issues. World Journal of Diabetes, v.8, n.II, p. 464-474, 2017.

MAGNO, Felippe Castro et al. Nutritional profile of patients in a multidisciplinary treatment program for severe obesity and preoperative bariatric surgery. ABCD, arq. bras. cir. dig., v.27, n.I, p.31-34, 2014.

MEHAFFEY, J.H.; MEHAFFEY, R.L.; MULLEN, M.G.; et al.Nutrient Deficiency io Years Following Rouxen-Y Gastric Bypass: Who's Responsible? Obes Surg., v.36, n.5, p.413-419, 2017.

MISCHLER, R. A.; ARMAH, S. M.; CRAIG, B. A. Comparison of Oral Iron Supplement Formulations for Normalization of Iron Status Following Roux-EN-y Gastric Bypass Surgery: a RandomizedTrial. Obesity Surgery, v.27, n.2, p. 424-431, 2017.

MOLEIRO, J.; MÃO DE FERRO, S.; FERREIRA, S. Efficacy of Long-Term Oral Vitamin Bi2 Supplementation after Total Gastrectomy: Results from a Prospective Study. Portuguese Journal of Gastroenterology, v.2, n.24, p. 24-31, 2019.

MONTANO-PEDROSO, J.C.; GARCIA, E.B. Postoperative intravenously administered iron sucroseversus postoperative orally administered iron to treat post-bariatric abdominoplasty anaemia (ISAPA): the study protocol for a randomised controlled trial. Trials, v.17, n.196, p.I-II, 2020.

MOREIRA, M.A.; ESPÍNOLA, P.R.M.; AZEVEDO, C.W. Intolerâncias alimentares e sintomas associados em pacientes submetidos à técnica de Fobi-Capella sem anel gástrico. ABCD, Arq. Bras. Cir. Dig., v.28, n.I, p.36-39, 2015.

OZATA M, MERGEN M, OKTENLI C, et al. Increased oxidative stress and hypozincemia in male obesity. Clin Biochem. 2002; 35(8):627-31.

PORIES, W.J.; SWANSON, M.S.; MACDONALD, K.G., et al. Who would have thought it? Na operation proves to be the most effective therapy for adult-onset diabetes mellitus. Ann Surg., v.7, n.22, p.339-52, 2015.

PIEGAS, L.S.; AVEZUM, A.; PEREIRA, J.C.; et al. Risk factors for myocardial infarction in Brazil. Am Heart J, v.23, n.146, p.331-338, 2015.

PUTTINI, Silvana. Obesidade: tratamento cirúrgico. Rev. Nutrição, v.4, n.17, 2014.

REPETTO, G.; RIZZOLLI, J.; BONATTO, C. Prevalência, riscos e soluções na obesidade e sobrepeso: Here, There, and Everywhere. Arq Bras Endocrinol Metab., v.47, n.6, p.633-635, 2014.

RIBOT J, FELIPE F, BONET ML. Changes of adiposity in response to vitamin a status correlate with changes of PPAR [gamma] 2 expression. Obesity. 2001; 9(8):500-9.

ROLIM, F. F.; CRUZ, F. S.; CAMPOS, J. M.; et al. Repercussões em longo prazo da derivação gástrica em Y de Roux em população de baixa renda: avaliação após dez anos de cirurgia. Revista Do Colégio Brasileiro de Cirurgiões, v.45, n.4, p.10-19, 2018. 
SABISTON JÚNIOR, Diego Carvalho. Tratado de cirurgia: as bases biológicas da prática cirúrgica moderna. Rio de Janeiro: Guanabara Koogan; 2015.

SEGAL, A.; FANDIÑO, J. Indicações e contra indicações para a realização das operações Bariátricas. Rev. Bras. Psiq., v.24, n.3, p. 68-71, 2016.

SILVA, A.L.; GOMES, C.G.O. Assessment of the gastro-jejuno-duodenal transit after jejunal pouch interposition. $A B C D$, arq. bras. cir. dig., v.28, n.4, p.231-233, 2015.

ZULET M, PUCHAU B, HERMSDORFF $\mathrm{H}$, et al. Vitamin A intake is inversely related with adiposity in healthy young adults. J Nutr Sci Vitaminol. 2008; 54(3):347-52. 